\title{
Entretien avec Reinhard Flender, directeur de Peermusic Classical
}

Interview with Reinhard Flender, director of the Peermusic Classical

Interview met Reinhard Flender, directeur van de Peermusic Classical

\section{Reinhard Flender}

\section{OpenEdition}

Journals

\section{Édition électronique}

URL : http://journals.openedition.org/temoigner/1248

DOI : $10.4000 /$ temoigner. 1248

ISSN : 2506-6390

\section{Éditeur :}

Éditions du Centre d'études et de documentation Mémoire d'Auschwitz, Éditions Kimé

\section{Édition imprimée}

Date de publication : 1 octobre 2014

Pagination : $38-39$

ISBN : 978-2-84174-674-3

ISSN : 2031-4183

\section{Référence électronique}

Reinhard Flender, «Entretien avec Reinhard Flender, directeur de Peermusic Classical», Témoigner. Entre histoire et mémoire [En ligne], 118 | 2014, mis en ligne le 01 octobre 2015, consulté le 23 octobre 2020. URL : http://journals.openedition.org/temoigner/1248 ; DOI : https://doi.org/10.4000/temoigner 1248 


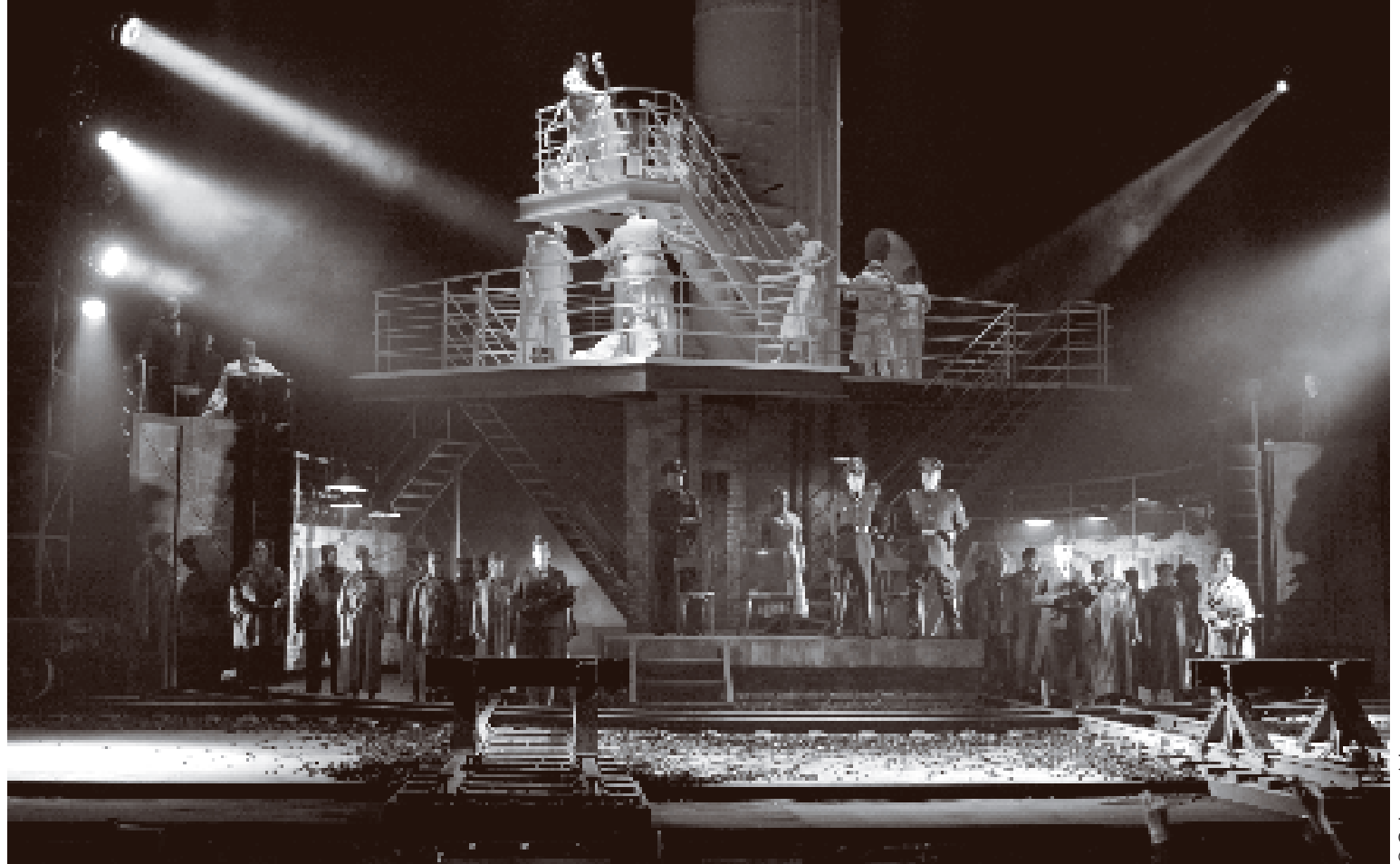

\section{LA PASSSAGÈRE, UN OPÉRA}

OPERA SORTI DU PURGATOIRE

Dans le précédent numéro de Témoigner nous avons consacré notre rubrique à L'Empereur de l'Atlantis de Viktor Ullmann, cette fois-ci, nous voudrions revenir sur un autre opéra, peu connu également La Passagère de Mieczyslaw Weinberg. Sa dernière représentation, à Londres, a suscité quelques débats.

$\rightarrow$ En association avec SResMusica
Ce qui est admirable, c'est la capacité de Weinberg de gérer le temps, la montée progressive de la tension, l'économie de musique permettant de marquer la difficulté du temps dans le camp, contrebalancée par des coups d'éclat orchestraux et vocaux qui permettent d'accrocher à l'œuvre.

mari, et nous fait revivre les épisodes de sa relation ambiguë et dominatrice avec la prisonnière.

Lors de la représentation du 20 septembre à Londres, 'est avec une troublante unanimité, du Guardian au Financial Times, del'Evening Standard au Tele graph, que les critiques anglais ont souligné la qualité pour mieux condan l'o pour mieux condanner musicalement lopéra. Lu estime que lœuvre est un « ratage total », quand un ancé t sur l'humanité ées pour décourager le mélomant avent rens: i trop de muiqu un un il n’y en pas assez pour intéresser le mélo to "il n'y en pas assez pour intéresser le mélomane! scène? Trop réaliste disent certains, trop théatralicé ju Trop realiste disent certains, trop theattralisée jugent les autres. Au fond, la presse anglaise port séfléchen la mö̈ (qui a toujours cefusé de monen réchéc du l bolchoi (qui a toujours refusé de monter cet opéra): louvre (qu convient pas «musicalement comment expliquer une telle cacophonie danalyses ? Effectivement, IActe I sinstalle lentement, de descendre (au propre comme au figuré) dans'enfer d’A scendre (au propre lexprotion psychologique et dram tiquprofond 'exploration psychologique d'ina drique, avec en Ce qui estadmirable, précisément, c'est la capacité de Ce qui est admirable, précisément, cest la capacité de de linberg de gérer le temps, la montée progressive marquer la difficulté du temps dans le camp, cont balancée par des coups d'éclat orchestraux et vocaux di, soutenant l'attention, permettent d'accrocher l'œuvre.

Á écouter et étudier la partition, Dimitri Chostakovitch, auteur de la Symphonie n ${ }^{\circ} 13$ Babi Yar et am de Weinberg, disait y avoir « mieux compris, à chaque fois, la beauté et la grandeur de cette musique ».

Oui,les camps de concentration mettentà lépreuve de la question même de l'irreprésentabilité de la vioence extrême, oui, il s'agit de concevoir un traitement particulièrement précis pour ne tomber ni dans le La force du livret lui permet d'éviter magistralement toute to est plus complexe et interessante que son diplomate de mari qui a la conscience bien propre et va finaletout oubler du passé de sa femme, de son pays, non pour pour elle, mass pour pressers sa propre reputation plus méprisable.

Dichell

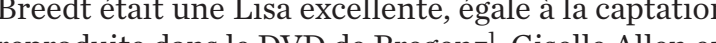
eprodite dans le DVD de Bregenz. Giselle Allen en son chant déchirant, quise fiche de la ujoliesser son pour atteindre lavérité de la musique joltude » du Son grand air '’ La direction de Sir Bichard Armstrong ten La direction le rythme sans brusquer la nécessaire len, soutenant è ra norre sans brusquer la nécessaire lenteur propre les décors. La nuse en scène de David Pornthey jeu constant de va-et-vient entre le pout du avecun jeu constant de va-et-rient entre le pont du paquebot

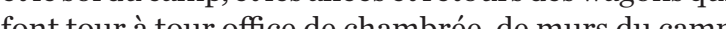
ou d'évoction des fours crématores.

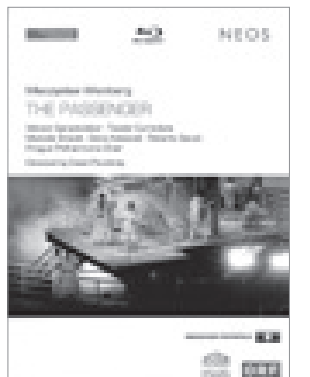

III (i) Disponible chez 
-. Ajout de lopéra par rapport au livre, le personnage dadeusz, le fiancé violoniste, apporte un climax dramatiqu à la finde lopera: sommé de jouer une valse

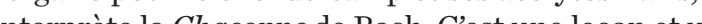
déf la défi lancé par lartiste, vrai détenteur, fut-il Juir, de the duiforne. Il paíra de sa ve la provocation. Ce thème du prison ceuxqula violent Il rappelle le Requiem der raconte Thistoire vraie du chef dorchestre Raphae Schàchter qui parvint à répéter et faire représenter de Verdi devant Eichmann au camp de

Ouvrage sur la culpabilité, l'incompréhension de soi comme des autres (Lisa semble sincere quand el e plaint auprès de son marícile était détestée de

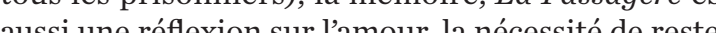
aussi úe réflexion sur lanour, la nécessité de rester dérisoire et suprême à la fois contre la barbare. Passagère est un vrai et pur moment d'opérarie. La Passagère est un vai at pur moment dopéra, magisth a lla attendu plus de quarante ans pour être joué, il hui faudra encore quelques anses ì lie pour ce quil est.

Ala findelarepresentation, une vieille dame élancée est venue saluer, franchissant précautionneusement les ralls.ZZofia Posmysz, lauteure du texte initial, qui a connu et a survécu à Auschwitz, qui a conn «Lisa». Oui, la Marta de lopéra est encore vivante, et qui ont souffert ne doivent pas être oubliés.

$$
\text { Jean-Christophe Le Toquin }
$$

Ce texte est une version adaptée et actualisée de la chronique
du to 20 septembre 2011 que Jean-Christophe Le Toquin avait fait paraitte

Pour la première fois, cet opéra a été représenté le jeudi 10 juillet
au Lincoln Center de New York: http://culture.pl/en/article/operatic-
holocaust-onstage-rouses-new-york

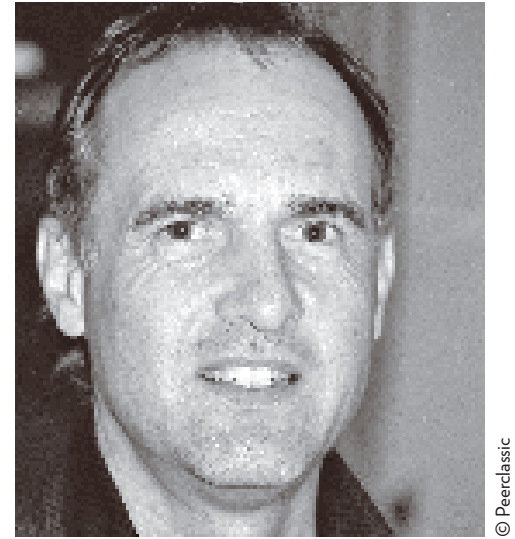

\section{Entretien avec..}

Reinhard Flender, directeur de Peermusic Classica

Peermusic Classical est la branche musique classique européenne du groupe Peermusic fondé en 1928 et aujourd'hui le plus grand éditeur mondial de musique avec 600000 œuvres à son catalogue. ResMusica a rencontré son directeur Reinhard Flender, parlant un français parfait, à propos de La Passagère de Weinberg.

Mieczyslaw Weinberg fait aujourd'hui partie des compositeurs importants de votre catalogue. Comment de sa carrière à lépoque soviétique?

Reinhard Flender: C'est par le Quatuor Danel, en 1999, et le premier mer: Cest par le Quatuor Danel, en 1999, et le premier mouvement du Quatuor $n^{\circ} 7$. Je n'ai pas été très impressionné au début, car ce mouvement pris isolément ne permet pas de savoir qui est Weinberg. C'est avec le Quintette que j’ai décidé d'aller à Moscou. Trente œuvres avaient été publiées par Sikorski, et tout le reste était libre. Il fallait aller vite, car ça avait traîné deplis desá par les quatuors et le Quintette.

\section{Et La Passagère?}

Reinhard Flender : Ce n'est qu'ensuite qu'on a entendu parler d'un opéra avec un thème, comment dire... qui nous regarde, qui concerne les Allemands. J'ai été très impressionné par la partition, écrite avec une telle intensité. Et il existe aussi un enregistrement où Weinberg chante lui-même l'intégralité de l'opéra en jouant lui-même au piano. On comprend qu'il a tout dans la tête!

\section{Comment
Bregenz?} Reinhard Flender : J'ai fait une proposition à tous les opéras, y compris Bregenz. David Pountney, que je navais jamais rencontré, étaíle directeur de ce festiva faire l'opér apportez-moila purtition. C'est plus vard quejai compris que Pountney conn. Cest plus pays parlo vailléde manière perfectionniste, est dépensébeaucour l'opér. Il y avat à l'époque 17 CD de Weinberg il a touse. tous achetés et,en 2006, lopéra átéjouéen version de Illa aimé tout de suite.

\section{En raison de son livret?}

Reinhard Flender : Le fait que Marta [c'est-à-dire Zofia Posmysz, auteure du texte source, cf. supra] soit Zofia Posmysz, auteure du texte source, cf. supra] soit estine vivable, très riche, pas polémique. Ily a me de théories, Chostakovitch poun

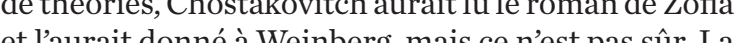
et Taurait donné à Weinberg, nais ce n'est pas sûr. La scène finale, ou Tadeusz jone la Chacone de Bach au Medvede Zofia n'en état pas trep contente, mais

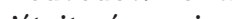
c'était nécessaire.

Weinberg a composé huit opéras, La Passagère étan le premier Quelle place cet opéra tient-il dansl'oeuvre du le premier. Quelle place cet opéra tient-Ida compositeur?

Reinhard Flender: On savait déjà que La Passagèr tout étit lí, tout était lié, tout ce quill a ecrit avant et après. Plus l'opéra unt ses mînise ines, plus on voit qu'ily a dans coper de Schnittre sur le polystylisme. On retrose le thème de la Leningrad de Chosto citations de Kurt Will Le plus révolion, et des citations de Kurt Weill. Le plus revolutionnaire, c'est dans les scènes des baraquements : quand les soldats

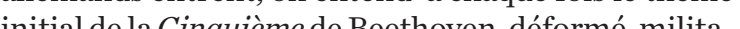

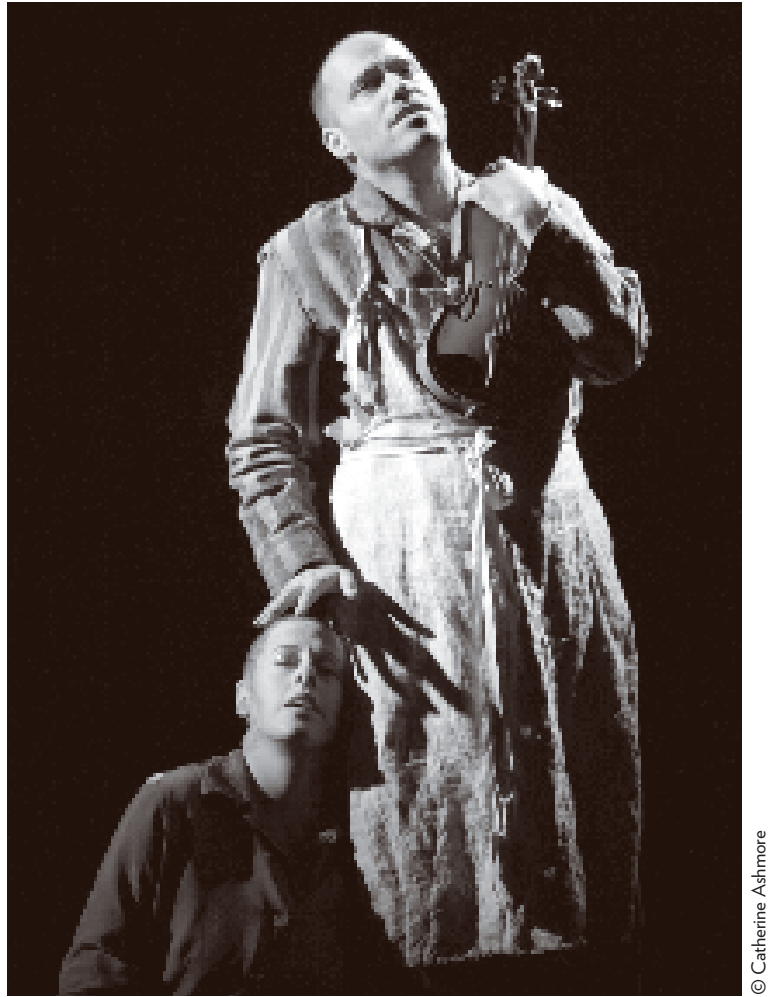

risé. Cette association systématique de l'armée et de Beethoven, c'est complètement fou... Si les musicien avaient conscience de cela, ils pourraient le rendre plus clairement. Il y a aussi beaucoup de citations, il fau cour Weinberg fait du recyclage.

L'œuvre a été jouée à Bregenz, Varsovie et désormais Londres. Y a-t-il eu des différences d'approche?

Reinhard Flender : Les musiciens anglais ont joué vec plus d'individualité, j’ai entendu des détails que je n’avais pas remarqués avant. C’était impressionnant.

\section{La suite est-elle déjà connue?}

(a) interprétation sera ( mivison d'opéraintéressé en n'ai pas réussia trouver de

Propos recueillis par Jean-Christophe Le Toquin
le 17 octobre 2011 